\title{
Adoption of Mobile Payments in Kenyan Businesses: A Case Study of Small and Medium Enterprises (SME) in Kenya
}

\author{
Erick O. Otieno \\ School of Computing and Informatics, \\ University of Nairobi,
}

\author{
Andrew M. Kahonge \\ School of Computing and Informatics, \\ University of Nairobi, Kenya
}

\begin{abstract}
This study focuses on adoption of mobile payment on business in Kenya. We employ Technology, Organization and Environment (TOE) to identify factors that motivate SME to adopt mobile payment methods in their businesses. To achieve this, we performed a cross-sectional survey and sampled 317 SMEs from three strata; hotels and restaurants, tours and travel and supermarkets. Structured Equation Model (SEM) analysis reveals a significant relationship between all indicators and latent variables; Technology, Organization and Environment. Based on these results, we then present a validated framework for the adoption of Mobile Payment platforms in SMEs.
\end{abstract}

\section{Keywords}

Mobile Payment, Structured Equation Model, Technology Organizational and Environmental Theory, Technology Acceptance.

\section{INTRODUCTION}

Over the recent years, mobile phones have played a very important role in the lives of many in the third world countries especially in Kenya with regards to mobile payment ranging from the banked to the non-banked, (Ahmad \& Yunos, 2012). Interaction between the clients and the merchants may take a variety of processes as indicated by (Toma, 2012). In developed countries, such as Japan mobile payment methods have taken forms of advanced chipsets in the mobile phones and Integrated Circuits Cards, an example being the "Wallet Mobile", (Toma, 2012).

\subsection{Background Information \\ 1.1.1. Electronic Commerce}

Electronic commerce (e-Commerce) has been variedly defined as the concept of trading online via the internet. This usually involves the buyers exchanging money and goods or services in a virtual environment even though the goods can either be virtually accessed online or delivered later on after the payment transactions have been made. E-commerce is defined by (Mutua, Oteyo, \& Njeru, 2013)as a form of electronic commercial engagement whereby transactions and selling of goods and services is done online through world wide web or through telecommunications network such as mobile telephone service provision. Also, a summary by, (Kinnuthia \& Akinusi, 2014) defines commerce as that transaction done over the internet. This therefore can be argued that there is a very thin line between $\mathrm{m}$-commerce and e-commerce.

According to (Jobodwana, 2009), m-commerce and ecommerce are a force to reckon with in Africa although mcommerce is perceived to in future surpass the e-commerce as a method of digital transaction. A study conducted on banks in Kenya by (Asiabugwa \& Munyoki, 2013) indicated a positive correlation between the adoption of e-commerce and performance. In their study, they concluded that from the result, banks that adopted e-commerce improved in their performance as opposed to those that did not. This indicates to us that e-commerce platforms are a good source of banks and to a greater extent Small and Medium Sized Enterprises' (SME) improved performance.

An indication is also given by (Mutua, Oteyo, \& Njeru, 2013)on the general trend of e-commerce adoption by SME in Kenya. In their study, they established that e-commerce was not widespread with about $43 \%$ of all the firms they surveyed having no functioning websites. Out of the sampled SMEs $31 \%$ of the firms had only static websites which could not interact with customers, while $22 \%$ of the firms had active websites that allowed the firm to interactive communication with customers. With this in mind, it can be argued that a substantial number of firms at $22 \%$ have e-commerce platform.

\subsubsection{Mobile Payment}

Mobile payment has been modelled in to four different domains by (Chaix \& Torre, 2012). These domains had been subdivided as; the operator-centred mode, the bank-centred model, the independent services model and the collaborative model, (Chaix \& Torre, 2012). The need to look at the mobile payment methods have been necessitated by lack of informative data on the various payment methods such as mobile payment and e-payments available, (Dahlberg, Mallat, Ondrus, \& Zmijewska, 2006)

\subsection{Problem Statement}

The question that could be asked is how would this contribute to the Small and Medium-sized Enterprises growth if adopted, and secondly, what would make the SME decision makers on E-Commerce platforms opt for the adoption of the mobile payment technology.

\subsection{Research Questions}

After the end of this study, the study needed to have answered the following research questions that emerge;

- What effect do technological, organizational and environmental variables have on the decision makers in firms to adopt or not to adopt mobile payment?

- What are the possible variables that decision makers in firms consider while opting not adopting mobile payment?

- Are there any other unknown possible extra reasons for decision makers in firms to adopt or not to adopt mobile payment?

\subsection{Rationale}

According to (Diniz, Vargas, \& Albuquerque, 2011), in their literature review work, it emerged that there were still missing gaps with regards to information relating to factors leading to 
adoption of mobile money technology at firm level. In their results, they indicated that literature available with respect to Consumer adoption took $30 \%$ of the literary work, with respect to Merchant adoption the literature available covered $4 \%$ and the technology factors covered only $3 \%$.

\section{LITERATURE REVIEW}

\subsection{Theories and Frameworks}

\subsubsection{Technology Acceptance Model}

The theory of Technology Acceptance Model (TAM) looks at factors that affect technology acceptance from an individual. For instance, it theorizes that an individual is prone to adopt a particular technology based on their attitude towards those technologies which in turn are driven by perceived usefulness and Perceived ease of use, (Bruner, Kumar, \& Clark, 2007). Even though the TAM has been widely quoted by many scholars in relation to technology acceptance, as applied by (Mbogo, 2010), it has equally received some critiques for dwelling mostly on factors emanating from an individual technology adoption point of view, (Bagozzi, 2007).

\subsubsection{Unified theory of acceptance and use of technology}

The use of Unified Theory of Acceptance and Use of Technology (UTAUT) in some of the information adoption research is an extension of TAM. It was discovered that (Venkatesh, Thong, \& Xu, 2012)incorporates more constructs of motivation, price value, and habit to the constructs of TAM namely attitude, perceived usefulness and perceived ease of use. The effects of the constructs on behavioural intention and technology use, it is hypothesized, are moderated by Individual differences such as age, gender, and experiences, (Venkatesh, Thong, \& Xu, 2012).

\subsubsection{Diffusion of Innovation}

The diffusion of Innovation looks at the rate at which new innovation is spreading, how the new innovation is spreading and why it is spreading in order to investigate the factors affecting the adoption of new information technology innovation both at individual and SME levels, (Yoon, 2009). The various factors to be looked into therefore are attached to both firm and individual's role in adapting to new technology.

\subsubsection{Technology, Organization and Environment (TOE)}

The theory of Technology, Organization and Environment (TOE) on the other hand looks at three major factors that are further broken down to smaller constructs when looking at how information technology is adopted at firm level, (Yoon, 2009), these factors include technological context within the SME, organizational context within the SME, and environmental context

\subsubsection{Organization Size}

The firm's capability with regards to financial as well as technical resources may be considered to positively or negatively influence how it makes decision on adoption of mobile payment technology. This leads to the following hypothesis:

H5: Larger organizational size leads to adoption of Mobile payment more than smaller Organizational size

\subsection{Conceptual Framework}

\section{Technological Context}

- $\quad$ Perceived benefits

- Operational unfriendliness due to non-real-time transactions

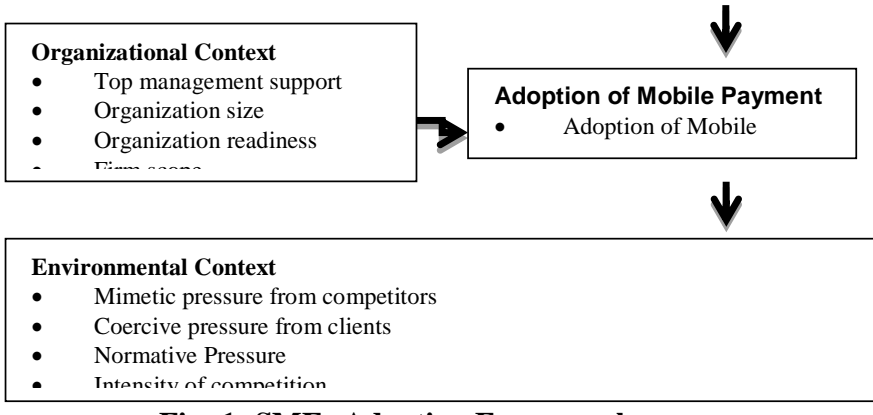

Fig: 1: SMEs Adoption Framework

\subsection{Research Hypothesis and Framework}

2.3.1. Technology factor, (Benefits, Operational friendliness, Security concerns)

2.3.1.1. Benefits of using mobile payment as a form of payment method in Small and Medium Enterprises

Every firm will try and analyze the cost of adopting a technology in verses the benefits the technology will accrue for the firm. In doing the cost benefit analysis, the firm may make a decision regarding the adoption of the technology. This led us to the following hypothesis.

H1: Perceived benefits of mobile payments lead to adoption of Mobile Payment

\subsubsection{Operational friendliness due to non-real- time transactions}

In real-time payment processes, the clients are supposed to get their requested product or services immediately without necessarily going through other steps such as going to the merchant or the SME. The same can be said also from the firm's point. This led us to the following hypothesis.

H2: Lower operational friendliness due to non-realtime transactions of mobile payments platform hinder adoption of Mobile Payment

\subsubsection{Security concern on Mobile Payment platform}

It was observed that (Yoon, 2009)applied the security concern in a study that was empirically investigating factors affecting organizational adoption. The study also had it as part of this study's construct this formulated the following hypothesis.

H3: Greater security concerns hinder the adoption of Mobile Payment

\subsubsection{Organizational readiness}

As indicated by (Yoon, 2009), organization readiness could be split in to two main constructs of financial and technical readiness. The organizational readiness, the study indicated could be separated as formative, and sub constructs derived from them. This research employed the same constructs and thus this study generated the following hypothesis.

H6: Higher organizational readiness leads to adoption of Mobile Payment 


\subsubsection{Firm scope}

Firm scope was also felt to be very vital since it could have a key indicator within the firm's profile. Firm scope has also been used as a construct by other researches such as (Yoon, 2009). In this research, respondents were on the issues ranging from local to global presence to define how the scope would affect their decision to adopt or not to adopt mobile payment platform.

H7: Greater firm scope leads to adoption Mobile Payment

\subsubsection{Environmental factor, (Mimetic pressure, Normativepressure, Client attitude, Competitive pressure)}

\subsubsection{Mimetic pressure}

According to (Yoon, 2009), mimetic pressure such as competition from other firms in the same market or otherwise could be an indicator of what could influence firms to adopt or not to adopt mobile payment platform. Thus this study came up with the following hypothesis.

H8: Greater mimetic pressure from competitors leads to adoption of Mobile Payment

\subsubsection{Client Attitude}

Intention to use a technology has been used as a predictor of actual usage of the technology at user's level of technology usage, (Venkatesh, Thong, \& Xu, 2012). The behavioural intention therefore has been found to significantly influence actual usage. (Yoon, 2009), added that client attitude can be said to constitute a form of coercive pressure. This leads to the following hypothesis:

H9: Greater coercive pressure from clients leads to adoption of Mobile Payment

\subsubsection{Normative Pressure}

Normative pressure, according to (Yoon, 2009), could be said to be pressures emerging from peer trade, professional, clients as well as supplier. (Yoon, 2009) went further to have sub constructs for the normative pressure during operationalization. This study thus had the following hypothesis.

H10: Greater normative Pressure leads to adoption of Mobile Payment

\subsubsection{Competitive pressure}

According to (Yoon, 2009) there is an established relationship between competitive pressures experienced by firms in an economic zone to the adoption of Information Technology. These relationships could either be financial or market pressure making them bow to pressure in either adopting or not adopting the information technology. This leads to the following hypothesis:

H11: Greater intensity of competition leads to adoption of Mobile Payment

\section{RESEARCH METHODOLOGY}

The Research applied the cross-sectional survey design. For qualitative research that gives generalizations in nature has been deemed appropriate to use Cross-sectional survey (Yoon, 2009), the questionnaire was sent to the respective decision makers within the SME.

Questionnaires have been successfully applied in qualitative research. As observed by (Kinnuthia \& Akinusi, 2014)questionnaire was a more objective option and relatively a quicker way to collect information. They also observed that questionnaire was an affordable way of collecting information emanating from a large group. Due to limited resources ant time, this study employed the questionnaire as a means of data collection.

It was noted that (Wanjau, Macharia, \& Ayodo, 2014) incorporated both open-ended and closed-ended questions in their studies to gather information. In supporting the reason to adopt the questionnaire as pointed earlier, some of the reasons they gave for the strategy was that the strategy was quicker to administer, it was none-biased from this study's point of view since it was above this study's influence and variability, in terms of cost it is much cheaper and it also gives the respondents ample freedom to respond without any prejudice or disadvantage of time constraints.

Structured Equation Model (SEM) has been applied in many scholarly works to analyze data using the Technology, Organization and Environment (TOE) platform to study factors affecting adoption of information technology by firms. The use of SEM emphasizes the usefulness of the TOE research model and theoretical framework for studying ebusiness (Zhu, Kraemer, Xu, \& Dedrick, 2004).

This study's unit of analysis was the Small and Medium Enterprises (SME) in Kenya, whereby a stratified random sampling technique was used in selected amongst a group of participating SME that fell in three major strata that have generally been perceived to be having a greater number of clients; Hotels and Restaurant, Tours and travel, Supermarkets and Retail outlets.

\subsection{The Stratified Sample Size Calculation}

In order to arrive at the Population of the target group, various databases were selected online that form professional or economic groupings of the said stratified groups such as Hotel owners and Keepers website database for hotels and restaurants, Tour Operators Associations website database for tours and travel and Business listing for Supermarkets and Retails business entities.

The choices of the databases was strategic in that they gave a view of countrywide, regional and in some cases global reach of the selected population target such that the study would eventually be considered representative.

For the Hotels and restaurants, the population was derived to be 218 registered, whereas the Tours and travel database indicated an estimated membership of 600 registered. Supermarkets and Retail database indicated an estimated total of 766 registered. This gave a total population of the target group as 1584 .This study took $20 \%$ of the target population to conduct the study on 317 SMEs in Kenya.

For purposes of this study and in an attempt to improve on accuracy in the data collection and analysis exercise, the target population was divided into three strata: (i) Hotels and restaurants; (ii) Tours and travel; and (iii) Supermarkets and retail. Stratification aims to reduce standard error by providing some control over variance. A study by (Mugenda \& Mugenda, 2003)indicates a sample size of $10 \%$ or $20 \%$ will be sufficient for a study. This study took $20 \%$ of the population to select a sample size of 317 of the study population. From each stratum the study proportionally used simple random sampling to select 317 respondents. The sampled size was proportionally specified using the formula below for each stratum sample size. 


$$
\frac{\mathrm{Ns}}{\mathrm{N}} \mathrm{Xnp} \quad=\mathrm{ns}
$$

Where:

$N$ is the overall population size, in this study 1,584 .

$n p$ is the overall sample size, in this study 317.

$n s$ is the stratum sample size being calculated.

Source: Sampling Essentials, (Daniel, 2012).

(i) Hotels and restaurants

$\begin{array}{lll}\frac{218}{1584} & \text { X } 317 & =44 \\ & \text { Tours and travel } \\ \frac{600}{1584} & \text { X } 317 \quad=120\end{array}$

(ii) Supermarkets and retail

$\frac{766}{1584} \times 317=153$

SMEs were then randomly selected from the three strata with respect to the target size shown above to attain the target overall sample size of 317 SMEs.

\subsection{Structured Equation Model}

In this study, the study constructs and their inter-relationship patterns were specified a priori. SEM was considered the best approach in the case of this study. Sample size, fit indices, standardized paths unidimesionality test and numerous other approaches were some of the key considerations that researches have been urged to take cognizant of, (Hoe, 2008).

In a research where there are many variations in any variables, SEM has been considered be used to test a modelled hypothesis by applying a linear equation system. Various kind of software was considered to be available which this research would have employed to generate iterations, goodness-of-fit and standard paths. For instance, (Hoe, 2008) suggest using software programs like EQS, while (Tobbin \& Kuwornu, 2011) applied the use of AMOS version 18 to test their research hypotheses. (Mayhew, Hubbard, Finelli, \& Harding, 2009), while using Structured Equation Model (SEM) to analyze their data also used different primary statistical software known as LISREL. All the software above have been successfully used, however, this research used the statistical software to test the validity of the hypothesized models as well as being employed to illustrate the Frequencies, means, standard deviations and the Structural Equation Model (SEM) analysis as well as the relationship between variables and the final analysis of the data therein.

\section{RESULTS AND DISCUSION \\ 4.1.Analysis of Response Rate}

From the 317 sampled respondents, 232 respondents representing the three categories of SMEs under this study filled and returned the questionnaires thus attaining a response rate of $73 \%$ this study achieved this through the use of an introduction letter which comprehensively explained the purpose of the survey, and constant reminders to the respondents via e-mail, phone calls and physical visits.

A majority of the respondents were from Supermarket and retail services and Tours and travel services with $43 \%$ and $42 \%$ respectively, and the least from Hotel and restaurant services with $15 \%$. This shows that all respondents were from the three SME industries under this study and therefore information received is sufficient for further analysis.

\subsection{Profiles of the SMEs under this study}

Section A of the questionnaire covered aspects of the annual revenue of the firm, the number of years the firm has been in business, number of employees employed by the firm and the IT operating budget as a percentage of the total generated budget.. Respondents were asked to indicate the estimated annual revenue generated by their respective firms. A majority of the respondents were from SMEs with estimated annual revenue of Sh. 5,000,001 to $10,000,000$ with $40.5 \%$ and the least with $3.9 \%$ did not have this information.

Regarding the SMEs annual operational budget as a per cent of the annual revenue, most firms' operational budget is more than $8 \%$ of the revenue with $50.9 \%$.

Respondents were also asked to indicate the number of years their respective SMEs have been in business since establishment.

Respondents also indicated the total number of employees working for their respective SMEs. Most SMEs employed 101 to 200 employees. The least of the respondents indicated working for SMEs that hire more than 400 employees.

The final question under this section required respondents to indicate the annual IT budget as a per cent of the firms' total annual budget.

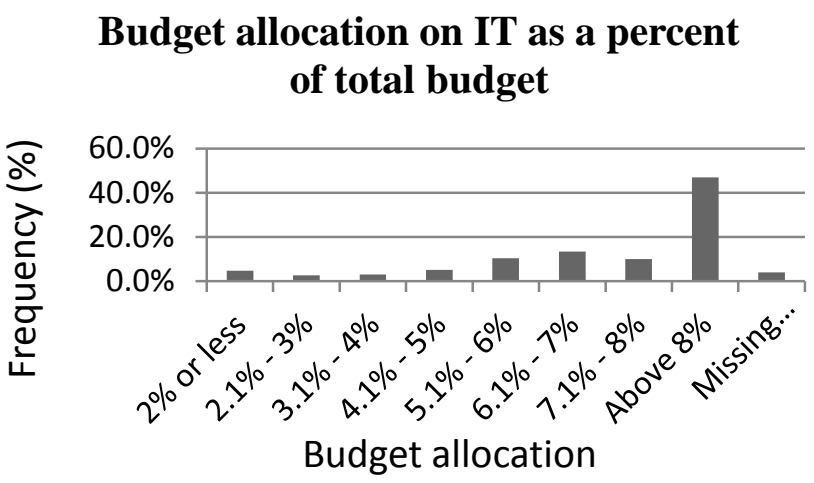

Fig 2: Distribution of SMEs by (\%) annual budget under IT

\subsection{Adoption of Mobile Payment status}

The status of adoption of mobile payment system by SMEs in the country was assessed from the data collected in section B of the questionnaire which required respondents to indicate their SMEs status of adoption. The respondents were to either select (i) our firm has already adopted mobile payment, (ii) our firm intends to adopt mobile payment or (iii) our firm does not intend to adopt mobile payment. 


\section{Adoption of Mobile payment system}

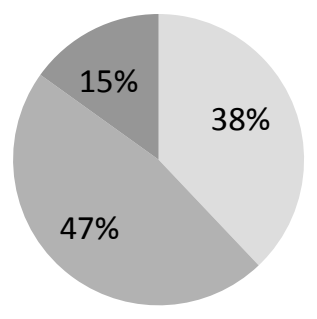

Mobile payment adopted

Planning to adopt

Not planning to adopt

\section{Figure 1: Adoption of mobile payment system in Kenya}

From this study's analysis as indicated in figure 3 above, most SMEs in Kenya are planning to adopt mobile payment systems with $47 \%$. The analysis also shows that only $15 \%$ of SMEs in the three industries are not planning to adopt the mobile payment systems.

\subsection{Adoption of Mobile Payment platforms by SMEs in Kenya}

\subsubsection{Technological factors}

Benefits of adopting mobile payment technology were assessed to determine if technological benefits affected adoption of the mobile payment platform. This section had 9 questions which were coded $\mathrm{t} 1, \mathrm{t} 2, \mathrm{t} 3, \mathrm{t} 4, \mathrm{t} 5, \mathrm{t} 6, \mathrm{t} 7, \mathrm{t} 8$ and $\mathrm{t} 9$. Data collected was analyzed using SEM model in STATA to illustrate regression and the correlation between the latent variables (benefits, friendliness and MgtSupport) and the observed variables ti where $\mathrm{i}=1$ to 9 as listed above.

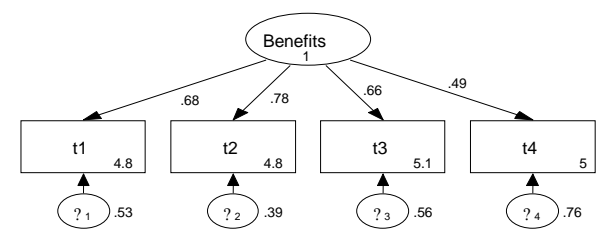

Fig 4: SEM model of effects of benefits of technology

With a p value of significantly less than 0.05 it was concluded that the latent variable (Benefits) is significant to explain all the four indicators, (Fig 4). To test for fitness of the model, this study used the chi square test and at $0.3921<0.5$ the model was thus adopted. The hypothesis as stated could therefore not be rejected.

H1: Perceived benefits of mobile payment lead to adoption of mobile payment.

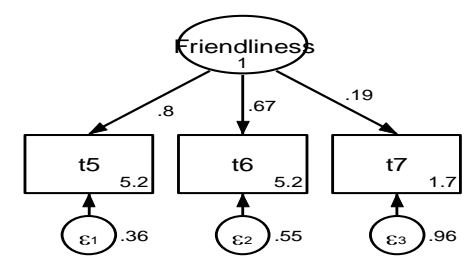

Fig 5: SEM model of effects of technological friendliness

With a $\mathrm{p}$ vale of less than 0.05 it was concluded that the technological friendliness is significant to explain all the three indicators t5, t6 and t7, (Fig 5). To test for fitness of the model, the study used the chi square test which was nil and thus fit. The hypothesis as stated therefore was not rejected.

$\mathrm{H}$ 2: Lower operational friendliness due to non-real-time transactions of mobile payments platform hinder adoption of mobile payment.

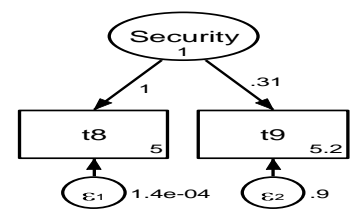

Fig 6: SEM model of effects of Security

Security concerns lower the adoption of mobile payment platforms. With a p vale of less than 0.05 security is significant unobserved variable to explain the two indicators t8 and $t 9$, (Fig 6). To test for fitness of the model, the study used the chi square test which was nil and therefore the model was adopted for further analysis of the hypothesis. This paper therefore could not reject the stated hypothesis.

H3: Greater security concerns hinder the adoption of Mobile Payment.

\subsubsection{Organizational factors}

Top managerial support, organization size and organizational readiness (coded as Mgtsupport, Size and Ready respectively) were assessed to test H4, H5 and H6. This section had 11 questions in sections $\mathrm{A}$ and $\mathrm{C}$. The codes adopted for the 11 questions were os 1, os 2, os 3, os $4, \mathrm{o} 1, \mathrm{o} 2, \mathrm{o} 3, \mathrm{o} 4, \mathrm{o} 5, \mathrm{o} 6$ and 07.Data collected was analyzed using means, frequencies and SEM model in STATA to illustrate regression and the correlation between the latent variables (organization size, top managerial support and organization readiness) and the observed variables osi where $i=1$ to 4 and oi where $i=1$ to 7 as listed above, and to test the significance of organizational factors to explain the observed variables.

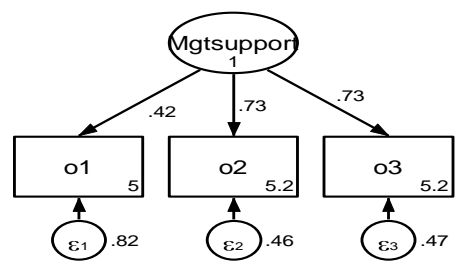

Fig 7: SEM model of effects of top managerial support

Fitness of the model was tested using chi square which was nil and thus adopted the model for further analysis. With a $\mathrm{p}$ vale of less than 0.05 the study concluded that top managerial support is significant to explain all the three indicators 01,02 and $\mathrm{o3}$, (Fig 7). The hypothesis as stated therefore was not rejecte

H4: Greater top management support leads to adoption of mobile payment.

From the filled questionnaires, $87 \%$ of SMEs which did not plan to adopt mobile payment platforms: i) have an annual revenue of less than Sh.500,000; ii) have an operational budget of less than $8 \%$; iii) have been operational for less than 10 years; and iv) hire less than 100 employees. With these observations on the profiles of the SMEs under this study as discussed in part 4.3 of this chapter, the following hypothesis therefore could not be rejected.

H5: Larger organizational size leads to adoption of Mobile payment more than smaller organizational size. 


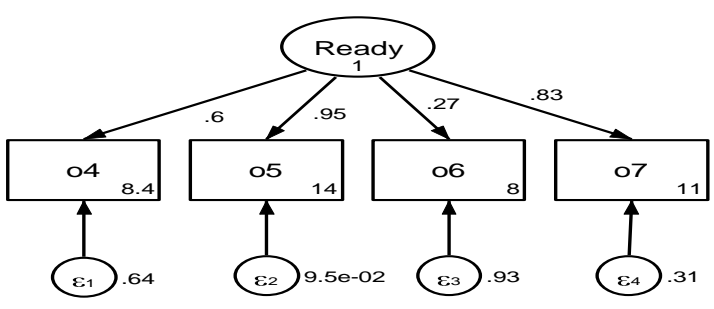

Fig 8: SEM model of effects of organizational readiness

Fitness of the model was tested using chi square which was at 0.0026 and thus adopted the model. With a $\mathrm{p}$ vale of less than 0.05 , it was concluded that higher organizational readiness is significant to explain all the four indicators $04,05,06$ and 07 , (Fig 8). The hypothesis as stated therefore was not rejected.

H6: Higher organizational readiness leads to adoption of mobile payment.

From the filled questionnaires, $85 \%$ of SMEs which do not plan to adopt mobile payment platforms only operate their business within Nairobi. With these observations on the profiles of the SMEs under this study as discussed in part 4.3 of this chapter, the study could not reject the following hypothesis.

H7: Greater firm scope leads to adoption of Mobile payment.

\subsubsection{Environmental Factors}

Pressure from the competition, clients and associations was assessed to test H8, H9, H10 and H11. This part of the questionnaire had 18 questions. The codes adopted for the 18 questions were ei with $i=1$ to 18 . Data collected was analyzed using means, frequencies and SEM model in STATA to illustrate regression and the correlation between the latent variables (mimetic pressure from competition, coercive pressure from clients, normative pressure, intensity of market pressure and firm scope) and the observed variables and to test for significance of the unobserved variable in explaining the observed variables. In the SEM models, these papers labelled the latent variables as Competition, Clients, Normative, Market and Scope respectively and are discussed below.

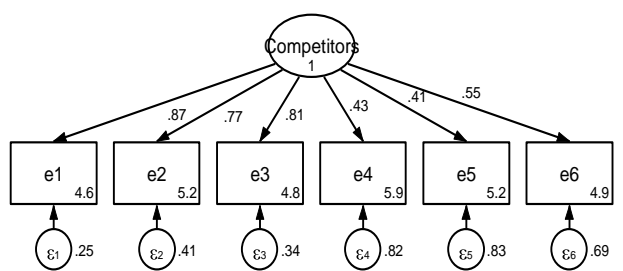

Fig 9: SEM model of effects of competition

Fitness of the model was tested using chi square which was at 0.2499 and the model was thus adopted. With a p vale of less than 0.05 across all the results, it was concluded that the latent variable (competitors) significant to explain all the six indicators, (Fig 9), and the hypothesis therefore could not be rejected.

H8: Greater mimetic pressure from competitors leads to adoption of mobile payment.

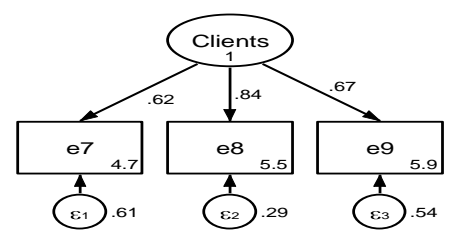

Fig10: SEM model of effects of pressure from clients

Fitness of the model was tested using chi square which was nil and thus the model was adopted. With a $\mathrm{p}$ value of less than 0.05 across all the results, it was concluded that the latent variable (coercive pressure from clients) was significant to explain all the six indicators, (Fig 10), and therefore the hypothesis could not be rejected.

H9: Greater coercive pressure from clients leads to adoption of Mobile Payment.

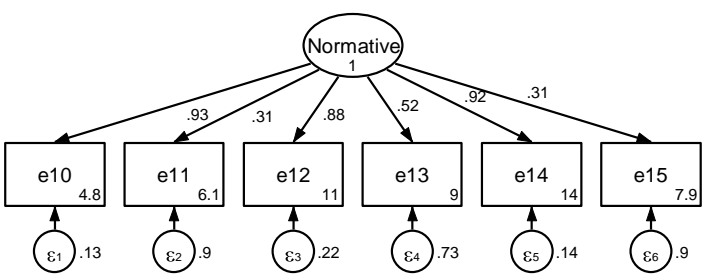

ig 11: SEM model of effects of normative pressure

Fitness of the model was tested using chi square 0.0001 and the model was thus adopted. With a p value of less than 0.05 across all the results, it was concluded that the latent variable (Normative pressure) is significant to explain all the six indicators, (Fig 11), and the hypothesis therefore could not be rejected.

H10: Greater normative pressure leads to adoption of Mobile Payment.

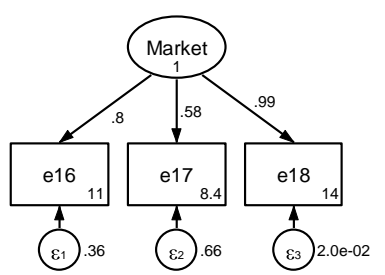

Fig 12: SEM model of effects of intensity of competition

Fitness of the model was tested using chi square which was nil and the model was thus adopted for further analysis. With a $\mathrm{p}$ value of less than 0.05 across all the results, it was concluded that the latent variable is significant to explain all the 3 indicators, (Fig 12) and the hypothesis therefore could not be rejected.

H11: Greater intensity of competition leads to adoption of Mobile Payment.

\subsection{SMEs with Plans to Adopt Mobile Payment Platforms}

Table 1: Target time for adoption of mobile payment by SMEs

\begin{tabular}{|l|r|r|}
\hline Target Time & Frequency & Percent (\%) \\
\hline Less than 6 months & 36 & $33 \%$ \\
\hline Above 6 - 12 months & 29 & $27 \%$ \\
\hline Above 12 - 18 months & 23 & $21 \%$ \\
\hline
\end{tabular}




\begin{tabular}{|l|r|r|}
\hline Above 18 - 24 months & 17 & $16 \%$ \\
\hline Information not available & 4 & $4 \%$ \\
\hline Not Applicable & 0 & $0 \%$ \\
\hline TOTAL & 109 & $100 \%$ \\
\hline
\end{tabular}

The study further did an analysis of the target dates for adoption of mobile payment systems by SMEs which were planning to adopt the technology. As indicated in table 1, 60\% of SMEs would have adopted the technology within the next 12 months although this projection is also reliant on the three factors analyzed under section 4.4 of this chapter.

\subsection{Additional factors that affect adoption of mobile payment platforms}

Respondents from SMEs which do not intend to adopt mobile payment platforms outlined factors that hinder them from adopting the technology. The outlined factors were grouped into the following three major categories: Poor marketing strategies by service providers; Lack of technical consultations by service providers; and Fear for loss of jobs, especially frontline staff, if the technology is adopted.

Respondents were also required to outline any other factors that would promote adoption of mobile payment platforms. The outlined factors were grouped into the following three major categories; Incentives from service providers; Free after sales service by service providers; and Intense marketing and training conducted by service providers.

\subsection{Discussion}

A majority of SMEs in Kenya regardless of the nature of business or size in terms annual revenue and number of employees are planning to adopt mobile payment systems within the next 12 months. This shows that despite the experienced factors as highlighted by $15 \%$ of the sampled SMEs as reasons to why they do not consider adopting mobile payment systems, most SMEs, $38 \%$ and $47 \%$ of SMEs have adopted and are planning to adopt mobile payment platforms. These $85 \%$ of the sampled SMEs have invested in IT personnel and systems in order to realize all the beneficial factors discussed in chapter four. All the hypotheses discussed in chapter three and tested under (i) technology, (ii) organization and (iii) environment as factors influencing adoption of mobile payment platforms could not be rejected since the latent variables generated from the above mentioned factors were strongly significant to explain all the observations indicated by the respondents. This implies that technology, organization and environment strongly affect adoption of mobile payments by SMEs in Kenya.

\subsection{Application of the study}

The application for this study is very significant and can be categorised in the firms' dimension, the mobile payment service provider dimension and policy making dimension.

\subsubsection{Firms' dimension}

It is evident that most firms feel that with the adoption of the mobile payment, there are prospects of increasing visibility and success within the competitive environment. It is therefore important that the firms consider looking at other prospects of encouraging their users to have mobile banking so as to encourage increase use of mobile payment.

\subsubsection{Mobile Payment service providers dimension}

With the response regarding security and interaction with mobile payment service providers, it is important that the service providers find ways of easing interactions between the firms who adopt mobile payments and their IT infrastructures. The service providers can look for ways of having secure Application Programmable Interfaces (API) that enable direct interactions and real-time transaction on mobile payments. This would increase the firms' interest in mobile payment.

\subsubsection{Stakeholder policy dimension}

This study also gives policy makers a food for thought on how best to draft regulatory framework that will guide the interactions between the Users, Firms and Service providers so as to allow conducive environment to uplift the Economy by increasing faster and secure mobile payment processes.

\subsection{Summarised Conceptual framework}

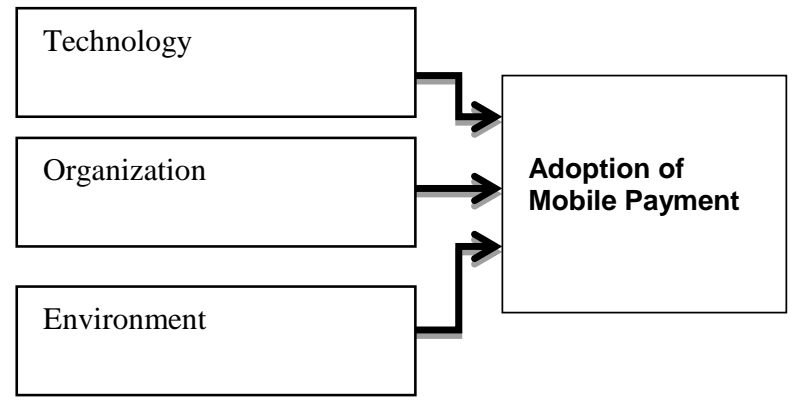

Fig 13: Summarised Concept for the adoption of Mobile Payment platforms by firms

The resulting conceptual framework therefore could be summarised as indicated in Fig 13 above where there is a technological, Organizational and Environmental influence on the decision to adopt or not to adopt mobile payment technology.

\section{CONCLUSIONANDSUGGESTIONS FOR FURTHER RESEARCH}

This chapter gives a summary of findings of the study, conclusions, suggestions for further research and recommendations to SMEs and service providers regarding the projected future of adoption of mobile payment platform in Kenya.

\section{CONCLUSION}

This case study of SMEs in Kenya on adoption of mobile payments aimed at researching on the effects of (i) technology, (ii) organization and (iii) environment on the status and rate of adoption of mobile payments. On successfully concluding the study, it was found out that indeed there is a greater correlation between the three construct and adoption of Mobile payment. This is a clear indication that indeed the Technology, Organization and Environment model (TOE) of Technology Adoption Model (TAM) is still applicable in firm setup. This paper therefore concluded that there was significant effect on various technology, environmental and organizational factors on the adoption of mobile payment if firms. 


\section{AREAS FOR FURTHER STUDY}

This study also brings into focus the need to explore using the mixed model of Technology Diffusion and TOE. It would be interesting to find out how the constructs from the two models would affect the mobile payment technology adoption.

\section{ACKNOWLEDGMENTS}

This study could not have been possible without the positive critiques and advisors who have contributed towards development of the paper.

\section{REFERNCES}

[1] R. Ahmad and Z Yunos, "The Application of Mixed Method in Developing a Cyber Terrorism Framework," Journal of Information Security, vol. 3, no. 3, pp. 209214, 2012, doi: 10.4236/jis.2012.33026.

[2] C Toma, "M-Payments Issues and Concepts," Informatica Economica, Academy of Economic Studies, vol. 16, no. 3, pp. 117-123, 2012, Availablefrom www.revistaie.ase.ro.

[3] J Mutua, I N Oteyo, and A W Njeru, "The Extent of ECommerce Adoption among Small and Medium Enterprises in Nairobi, Kenya," International Journal of Business and Social Science, vol. 4, no. 9, pp. 116-122, 2013, Available from http://www.ijbssnet.com.

[4] J N. K Kinnuthia and D M Akinusi, "The magnitude of barriers facing e-commerce businesses in Kenya," Journal of Internet and Information Systems, vol. 4, no. 1, pp. 12-27, 2014

[5] Z N Jobodwana, "E-Commerce and Mobile Commerce in South Africa: Regulatory Challenges," J. Int. Commercial Law Technol, vol. 4, no. 4, pp. 287-298, 2009, Available from http://www.jiclt.com/index.php/jiclt/article/viewFile/93/9 21.

[6] M A Asiabugwa and J Munyoki, "E-commerce Strategy and Performance of Commercial Banks in Kenya," African Journal of Business and Management, vol. 3, 2013, Available from http://www.aibuma.org/archive/journal/vol_three/PAPE R\%203.pdf.

[7] L Chaix and D Torre, "Which economic model for," in 23rd European Regional Conference of the International Telecommunication, Vienna, Austria, 2012, available at: http://hdl.handle.net/10419/60380.

[8] T Dahlberg, N Mallat, J Ondrus, and A Zmijewska, Mobile Payment Market and Research - Past, Present and Future Proceedings: Proceedings of Helsinki Mobility Roundtable, 2006, Available from http://sprouts.aisnet.org/6-48.

[9] E H Diniz, F G Vargas, and J P Albuquerque, "Mobile Money and Payment: a literature review based on academic and practitioner-oriented publications (20012011)," in Proceedings of SIG GlobDev Fourth Annual Workshop, Shanghai, China, 2011, Available from http://www.scribd.com/doc/93386441/24-REVISEDDiniz-Mobile-Money-and-Payment-Nov-14-2011.

[10] G C Bruner, S A Kumar, and T Clark, "Toward a Unified Theory of Consumer Acceptance Technology, Psychology \& Marketing," Wiley Periodicals, Inc, vol.
24, no. 12, pp. 1059-1084, 2007, http://dx.doi.org/10.1002/mar.20196.

[11] M Mbogo, "The Impact of Mobile Payments on the Success and Growth of Micro-Business: The Case of MPesa in Kenya," vol. 2, no. 1, 2010, Available fromhttp://www.ajol.info/index.php/jolte/article/view/51 998

[12] R P Bagozzi, "The Legacy of the Technology Acceptance Model and a Proposal for a Paradigm Shift," vol. 8, no. 4, 2007, Available from www.aisel.aisnet.org.

[13] V Venkatesh, J Y. L Thong, and X Xu, "Consumer acceptance and use of information technology: extending the unified theory of acceptance and use of technology," vol. 36, no. 1, pp. 157-178, February 2012

[14] Yoon, "An empirical investigation of factors affecting organizational Adoption of virtual worlds," Ph.D. dissertation 2009.

[15] K Sargent, P Hyland, and S Sawang, "Factors Influencing the Adoption of Information Technology in a Construction Business Kimberley," vol. 12, no. 2, 2012, Available fromhttp://epress.lib.uts.edu.au/journals/index.php/AJCE $\mathrm{B} /$ article/view/72.

[16] K Wanjau, N R Macharia, and E M. A Ayodo, "The magnitude of barriers facing e-commerce businesses in Kenya," vol. 4, no. 1, pp. 12-27, 2014.

[17] K Zhu, K L Kraemer, S Xu, and J Dedrick, "Information Technology Payoff in E-Business Environments: An International Perspective on Value Creation of EBusiness in the Financial Services Industry," vol. 21, no. 1, pp. 17-54, 2004, Available from http://www.jstor.org/stable/40398783.

[18] M Mugenda and A G Mugenda, Research Methods: quantitative and qualitative approaches. Nairobi: ACTS press, 2003

[19] J Daniel, Sampling Essentials.: Sage Publications, Inc, 2012, ch. Chapter 5: Choosing the type of probability sampling, pp. 131-132.

[20] S L Hoe, "Issues and procedures in adopting structural equation modelling technique," vol. 3 , no. 1, pp. 76-83, 2008, Available from http://jaqm.ro/issues/volume3 ,issue- $1 /$ pdfs/hoe.pdf

[21] P Tobbin and J K. M Kuwornu, "Adoption of Mobile Money Transfer Technology: Structural Equation Modeling Approach," vol. 3, no. 7, 2011, Available from www.iiste.org.

[22] M J Mayhew, S M Hubbard, C J Finelli, and T S Harding, "Using Structural Equation Modeling to Validate the Theory of Planned Behavior as a Model for Predicting Student Cheating," vol. 32, no. 4, pp. 441-468, 2009.

[23] S Gupta and $\mathrm{H} \mathrm{Xu}$, "Examining the Relative Influence of Risk and Control on Intention to Adopt Risky Technologies," vol. 5, no. 4, 2010. 